\title{
Infection of hepatitis $C$ virus genotypes in hepatocellular carcinoma patients from rural areas of Faisalabad region, Pakistan
}

\author{
Muhammad Nawaz ${ }^{1}$, Salma Siddique ${ }^{1,}$ Irfan Manzoor ${ }^{1}$, Shahid Nadeem ${ }^{1}$, Salah-ud-Din ${ }^{1}$, Hamid \\ Shabbir $^{2}$, Rahat Naveed ${ }^{1,3}$ and Muhammad Ibrahim Rajoka ${ }^{1 *}$ \\ ${ }^{1}$ Department of Bioinformatics and Biotechnology, Government College (GC) University, Allama lqbal Road, Faisalabad- \\ 38000, Pakistan. \\ ${ }^{2}$ Punjab Institute of Nuclear Medicine, Punjab Medical College Road, Faisalabad, Pakistan. \\ ${ }^{3}$ Department of Zoology, GC University, Allama Iqbal Road, Faisalabad-38000, Pakistan.
}

Accepted 17 June, 2011

\begin{abstract}
The aim of this retrospective study was to investigate the infection of hepatitis $\mathrm{C}$ virus (HCV) genotypes in hepatocellular carcinoma (HCC) patients from rural areas of Faisalabad region. Among 179 HCC subjects, men and women were 51 and $49 \%$, respectively. All samples positive for HCV RNA by qualitative PCR were genotyped, applying genotype-specific PCR. They were confirmed using HCV 5' non-coding region sequence analytical data. Major risk factor for HCC development and progression was identified to be chronic HCV. It was found among $56.5 \pm 2.1$ years of age. All these HCC cases were HCV-related and no case was linked with other types of viruses. Using genotype specific primers, HCV genotype 3a $(55.3 \%)$ was significantly $(\mathrm{P}<0.0001)$ higher, followed by $3 \mathrm{~b}(15.8 \%), 1(9.24 \%), 4(8.05 \%)$ and $2(7.15 \%)$. Other genotypes, namely $5 \mathrm{a}$ and $6 \mathrm{a}$ were only 1.19 and $1.05 \%$ among the HCC patients. About $1.05 \%$ remained un-typed because of minute viral load. HCV genotype 3a was strongly linked with HCC, followed by $3 \mathrm{~b}$. Moreover, HCC was linked with liver cirrhosis (81\%). It is suggested that genotyping may be recommended before starting interferon therapy.
\end{abstract}

Key words: Etiology, genotyping, hepatitis $\mathrm{C}$ virus, hepatocellular carcinoma, prevalence, $\alpha$-fetoprotein.

\section{INTRODUCTION}

There are about 200 million patients with hepatitis $C$ virus $(\mathrm{HCV})$ in the world and three to four million individuals are diagnosed as new cases every year (Farshapour et al., 2010). Approximately 2.0, 2.7, 5.0 and 10.0 million cases are in Japan, the United States, Europe and Pakistan, respectively (Higuchi et al., 2002). HCV is a formidable cause of morbidity and mortality worldwide and has higher propensity for causing liver disease, leading to chronic hepatitis $\mathrm{C}$. Chronic HCV infection may cause liver cirrhosis and hepatocellular carcinoma (HCC) over the course of 20 to 30 years if not cured timely. The incidence of HCV-related HCC is increasing tremensdously

*Corresponding author. E-mail: mibrahimrajoka47@gmail.com. Fax: +92 419201419 . in Europe, Japan, North America, Pakistan and Taiwan (Hamid et al., 2004; Kao and Chen, 2002). Thus, there is a dire need to understand the risk factors for HCC development and progression and most essentially to provide effective treatment for HCV in time to prevent its progression to the end-stage, that is, liver diseases and HCC.

It is surmised that HCV genotypes may be related to disease progression. Therefore, further understanding of the clinical relevance and therapeutic implications of the HCV genotypes is crucial for designing personalized therapies for patients with chronic HCV infection. Recent molecular epidemiological studies have documented marked differences in genotype distribution by geographical regions and among different patient groups. Based on genetic differences between HCV isolates, the heaptitis $\mathrm{C}$ virus is classified into six genotypes (Farshapour et 
al., 2010, Simmonds et al., 2005) with several subtypes within each genotype (represented by letters). Genotypes 1,2 and 3 are widely distributed (Farshapour et al., 2010) throughout the Asian countries (Japan, Taiwan, Thailand, India, Pakistan and China), Australia, Europe and the USA. Genotype 4 is predominantly confined to the Middle East, Central Africa and Egypt (Higuchi et al., 2002). Genotypes 5 and 6 are found mainly in South Africa and South East Asia, respectively (Hamid et al., 2004). The highest prevalence of HCV has been reported from Egypt (Frank et al., 2000).

The global epidemiology of viral hepatitis $A$ and hepatitis B is well established, but HCV data related with HCC remain limited in Pakistan (Tong et al., 1996; Kao and Chen, 2002; Hamid et al., 2004) because of expenses involved in diagnosis. Determination of $\mathrm{HCV}$ genotypes is of clinical significance in assessing the drug responsiveness and prognosis of the HCC patients. Moreover, the HCV genotypes have epidemiologic significance as well, as they indicate transmission route of infection and have not received extensive studies in Pakistan, partly due to the expenses involved and partly due to practical difficulties involved in getting samples for the detection of HCV RNA in the serum of infected individuals. Further, large scale and long term follow-up studies are necessary to clarify the relationship between genotype and disease progression in different areas (Waheed et al., 2009) of each country. Other risk factors for disease progression and development of HCC are age at infection, diabetes, gender, hepatic fibrosis (particularly cirrhosis), host genetic factors, iron overload, greater degree of hepatic inflammation combined with steatosis, and viral load with its quasi-species (Waheed et al., 2009). In addition to these, other potential risk factors may include co-infection with HBV, or human immunodeficiency virus (HIV), smoking and obesity (Idrees et al., 2008; 2009). Peripheral viral load and viral genotypes were also reported to have impact on progression of disease. However, other authors found no association of the development of HCC with a particular HCV genotype (Ryu et al., 2009). Dissimilar genotypes of $\mathrm{HCV}$ have relation with epidemiological studies, clinical management of HCV infection, response rate to anti-viral treatment and effective vaccine development

Patients living in rural areas cannot afford expensive medical checkups and go to the hospital only when their illness gets aggravated and become very weak. This study was carried out to find out the infection of HCV genotypes in HCC patients in rural population, association between particular HCV genotypes and HCC and suggest some strategic intervention for the control of this mortal disease.

\section{MATERIALS AND METHODS}

\section{Study design}

This retrospective study was designed and conducted at PINUM from January 2006 to December 2009 (356 patients) in order to determine the true prevalence of $\mathrm{HCC}$ and chronic HCV and their possible association. Of these subjects, 179 satisfied the criteria for inclusion in this study: HCC was confirmed by liver imaging using ultra-sonography and computed tomography or histologically or chronic HCV was of any etiology. The diagnosis of liver cirrhosis was done mainly based on the histological evidence. All clinical records of these patients were checked to judge the etiology of HCC. Histological observations of liver tissue surrounding the cancer were also documented. Possible sources of transmission of disease and duration of blood-borne infectious hepatitis were also recorded. For calculation of time of transmission of HCV infection, time of first blood transfusion or first major/minor surgery was also recorded. Data on possible community routes of infection such as cautery, circumcision and tattooing as well as potential medical routes of infection such as history of taking different injections, history of previous hospitalization and surgical operations; dental procedures (e.g., tooth extraction/filling and gum treatment), and other invasive medical procedures (e.g., intravenous catheterization, abscess drainage, esophageal vortices, scleratherapy, pulmonary endoscopy, urinary catheteri-zation, upper or lower gastrointestinal endoscopy) were also recorded.

Married women were also asked about their history of obstetric and gynecological interventions (e.g., cautery, curettage and dilatation, evacuation operations, healthcare provider, mode of delivery, place of delivery and handling person/s) as described previously (Frank et al., 2000). The research described in this work followed the tenets of the Declaration of Helsinki promulgated in 1964 and was approved by the institutional human experimentation committee and PINUM.

After informed consent, the subjects were asked to provide a blood sample for HCV testing during their visits to PINUM. All blood samples were screened for anti-HCV, anti-HBc, HBsAG, antiHBsAg, anti-HIV 1 and HIV 2 using ELISA kits (DRG Instruments, Germany) following the instructions of the manufacturers. Serum hepatitis B surface antigen ( $\mathrm{HBsAg}$ ) antibody or anti-HCV were used as the positive markers of chronic viral hepatitis infection.

\section{Laboratory testing}

$10 \mathrm{ml}$ of venous blood was collected from all the study subjects and sera were tested for liver function tests and HCV $\operatorname{lgG}$ antibody using antibody kit (Innogenetics, Ghent, Belgium). All respondents having $\mathrm{ALT}$ more than $100 \mathrm{IU} / \mathrm{L}$ were tested through ultrasonography and computed tomography.

HCV samples collected from the respondents and their family members were also analyzed for HCV RNA using a previously validated, in-house-nested reverse transcriptase polymerase chain reaction as described previously (Kao and Chen, 2002). Fresh plasma samples, processed within $2 \mathrm{~h}$ of collection by venous puncture, were stored at $-70^{\circ} \mathrm{C}$, after the addition of guanidine isothiocyanate RNA extraction buffer.

\section{Serological analysis}

All serological tests were performed as described previously (Idrees et al., 2009) using standard kits, following the instructions of the manufacturers. Liver function tests namely alanine amino trasnferase $(A L T)$, aspartate amino transferase (AST), alkaline phosphatase (ALP), $\gamma$-glutamyl transpeptidase (GGT) and bilirubin levels of all patients were determined using an auto-analyzer as described previously (Bukhari et al., 2010; Idrees et al., 2009). Serum $\alpha$-fetoprotein (AFP) concentration was estimated by chemiluminescent immunometric commercial diagnostic kit using immunoassay system (Diagniostic Products, Los Angeles, CA, USA). 
Table 1. Serological parameters* and etiology of HCC patients $(n=179)$ and chronic HCV control subjects $(n=177)$.

\begin{tabular}{lll}
\hline Serological parameter & Value in HCC patient & Value in chronic HCV patient \\
\hline Female & $88(49)$ & $88(49.7)$ \\
Age \pm SD (year) & $55 \pm 2.2(51$, male) & $35.5 \pm 5.5(50.3)$ \\
ALT (IU/L) & $58 \pm 3.2(49)$ & $36.1 \pm 4.5(49.7)$ \\
AST (IU/L) & $>60(49.0)$ & $\approx 42 \pm 6.5(100)$ \\
ALP $(I U / L)$ & $>62(40.2)$ & $\approx 40 \pm 3.5(100)$ \\
AFP $(\mathrm{ng} / \mathrm{L})$ & $150(100)$ & $37.5 \pm 4.5(100)$ \\
GGT (U/L) & $400(99.2)$ & $50 \pm 2.5(100)$ \\
Bilirubin (mg/dL) & $126.7(65.2)$ & $65 \pm 4.5(100)$ \\
Anti-HCV positive alone & $>1.5(100)$ & $0.5 \pm 0.05(99)$ \\
HCV-RNA PCR +ve & $176(98.3)$ & $177(100)$ \\
HIV & $169(94.4)$ & $170(96)$ \\
Cirrhosis present & $0(100)$ & $0(100)$ \\
\hline
\end{tabular}

${ }^{*}$ The serological predictors were all elevated than the cutoff value for each parameter.

ALT, Alanine aminotransferase; AST, aspartate aminotransferase; ALP, alkaline phosphatase; AFP, $\alpha-$ fetoprotein; GGT, $\gamma$-glutamyl transpeptidase. The values in parenthesis are expressed in percentages.

\section{Qualitative and quantitative detection and genotyping of HCV RNA}

RNA extraction was carried out employing a commercial viral RNA isolation kit (Qiagem, Germany) following the instructions of the manufacturers. HCV-RNA was transcribed into cDNA immediately using primers (see below). For molecular identification of HCV, nested-RT qualitative characterization was done. The following sets of primers from the $5^{\prime}$ untranslated region of the viral genome were used to amplify the HCV RNA45: P1 (outer antisense): 5'CGACACTCCACCATAGAT-3'; P2 (outer sense), 5' GCTCATG GTGCACGGTCTA-3'; P3 (inner antisense): 5'-GTG CTGTGAGG AACTACTGTCT-3'; and P4 (inner sense): 5'- ACTCGCAAGCA CCCTATCA -3'.

The primers and oligoprobes gotten were synthesized from Applied Bio-systems, USA. The amplified products were electrophoresed in a $1 \%$ agarose gel, stained with ethidium bromide, and recorded on a digital imager (Eagle Eye II, Stratagene, LaJolla, CA). Sequence analysis of amplified products was performed by a commercial company (Fermentas, Pakistan). Sequences of candidate isolates were aligned with representative sequence of each major genotype or subtype selected from the NCBI GenBank database. Phylogenetic analysis of HCV isolates was performed by constructing phylogenetic trees using the neighbour-joining method, using the bootstrap re-sampling test. HCV RNA was quantitatively determined using a real-time PCR employing HCV RNA quantitative kit (Roche Amplicor HCV Monitor test kit) according to the suppliers' instructions.

\section{Statistical analysis}

Statistical analysis was carried out for the study using GraphPad Instat3.0 software; free downloadable from the internet.

\section{RESULTS}

Biochemical, demographic and clinical data of patients are presented in Table 1. 179 respondents who tested HCV seropositive and HCV-RNA PCR-positive at enrollment, were included in this analysis. A total number of 91 $(51.0 \%)$ were males, and 2 were aged $24 \pm 10$ years. Overall, $99.9 \%$ were aged $56.5 \pm 2.1$ (Table 1). We used 177 subjects as chronic HCV controls who also tested HCV sero-positive on enrolment. Their demographic, biochemical and clinical data are shown in Table 1.

All HCC patients had elevated level of serum bilirubin $(>14.2 \mu \mathrm{mol} / \mathrm{L})$ and ALP $(>150 \mathrm{IU} / \mathrm{L})$. In all the patients with HCC, ALT (>60 IU/L) and AST (>62 IU/L) levels were abnormal in 49.0 and $40.2 \%$ of HCC patients. AFP and GGT levels were abnormal in 99.2 and $65.2 \%$ patients suffering from HCC, respectively. Hepatic cirrhosis was found in $81 \%$ patients with HCC. All the patients were negative for anti-HIV 1 and anti-HIV 2 on enrollIment. All HCC patients had significantly higher values of all serological parameters and it is a normal trend.

Among the 179 patients suffering from HCC, HCV antibodies were detected in 176 (98.3\%) serum samples. Three patients were found tissue-positive by PCR but they did not carry anti-HCV antibodies. Of these HCC patients with chronic $\mathrm{HCV}, 51 \%$ were male and $49 \%$ were females. All the HCC patients had past history of chronic HCV infection. 21 of them had also cholecystitis. Load of peripheral HCV RNA varied from $2.5 \times 10^{3}$ to $5.4 \times 10^{7}$ copies $/ \mathrm{ml}$. We did not find significant difference $(P<0.235)$ between HCV load in serum samples and tissue samples of the same HCC patients. Also, neither from PCR analysis nor from anti-bodies or serological records, could we find any etiological information about the two patients, and they remained un-typed. They fell in the age range of $24 \pm 10$ years. This infection did not follow general crite- 
Table 2. Distribution of HCV genotypes in HCC men and women patients.

\begin{tabular}{lccc}
\hline Genotype & Men & Women & Total $(\%)^{\star}$ \\
\hline 3a & 49 & 50 & $99\left(55.30^{\mathrm{a}}\right)$ \\
3b & 13 & 15 & $28\left(15.80^{\mathrm{b}}\right)$ \\
1 & 7 & 9 & $16\left(9.24^{\mathrm{c}}\right)$ \\
4 & 6 & 8 & $14\left(8.05^{\mathrm{c}}\right)$ \\
2 & 6 & 7 & $13\left(7.15^{\mathrm{c}}\right)$ \\
5a & 1 & 2 & $3\left(1.34^{\mathrm{d}}\right)$ \\
6a & 1 & 2 & $3\left(1.19^{\mathrm{d}}\right)$ \\
Un-typed & 1 & 2 & $3\left(1.05^{\mathrm{d}}\right)$ \\
\hline
\end{tabular}

Values given in parenthesis followed by different alphabets differ significantly with the application of Tukey Krammer test using Grapgpad Instat3 software; ${ }^{*} \mathrm{P}<0.0001$.

ria of HCC development and progression.

HCV genotyping of the 179 patients was performed using standard technique (Idrees et al., 2009). The predominant genotype was genotype $3(3 \mathrm{a}=56.18 \%$, $3 \mathrm{~b}=$ $15.80 \%)$ followed by genotype $1(9.24 \%), 4(8.05 \%), 2$ $(7.15 \%), 5 a(1.34 \%)$ and $6 a(1.19 \%)$, respectively (Table 2). Most hepatitis $C$ infections in Pakistan are caused by HCV genotypes $3 \mathrm{a}(56.18 \%)$, 3b (15.80\%), followed by 1 $(9.24 \%), 4(8.05 \%), 2$ (7.15\%), 5a (1.34\%) and 6a $(1.19 \%)$ (Table 2).

\section{DISCUSSION}

Epidemiological studies in different countries of the world exhibit a wide variation in HCV prevalence pattern, though incidence of HCV development and progression is higher among less developed nations (Idrees, 2008; Idrees et al., 2009). Chronic HCV has been identified as the major risk factor for the development of HCC. Several viral and host factors linked with HCC development have been studied extensively in recent years (Farshapour et al., 2010). We found that longer life and male gender were significantly more linked with HCC development (Table 1). This association between HCC and donor age and gender was also observed in other countries (Tavares et al. 2004; Idrees et al., 2009). This suggests that older population with longer times of exposure to the various infection risk factors have a greater propensity of being victim of chronic HCV leading to HCC.

All HCC patients had elevated level of serum bilirubin $(>14.2 \mu \mathrm{mol} / \mathrm{L})$ and ALP (>150 IU/L), ALT (>60 IU/L) and AST (>62 IU/L). AFP (99.2\%) and GGT (65.2\%) levels were abnormal in patients suffering from HCC and it is a normal trend. Hepatic cirrhosis was found in $81 \%$ patients with HCC. All the patients were negative for anti-HIV 1.

All HCC patients had past history of chronic HCV infection. 21 of them also had cholecystitis. Load of peripheral HCV RNA varied from $2.5 \times 10^{3}$ to $5.4 \times 10^{7}$ copies $/ \mathrm{ml}$. We did not find significant difference $(P<0.235)$ between HCV load in serum samples and tissue samples of the same HCC patients. Neither from PCR analysis nor from anti-bodies or serological records, could we find any etiological information about two patients, and it remained un-typed. They fell in the age range of $24 \pm 10$ years. This HCC infection did not follow general criteria of HCC development and progression as mentioned earlier.

Several host and viral factors have been involved in the onset of HCC. Among the viral factors, it has been reported that the presence of HCV genotypes are among the risk factors for HCC. We found that older patients had HCC. This finding is consistent with earlier studies where it was reported that it took several years for the onset of HCC in HCV patients (Idrees et al. 2009). AFP elevation was observed in $99.2 \%$ patients with HCC, while cirrhosis was present in $81 \%$ cases (Table 1) among the HCC patients studied. Earlier studies reported that cirrhosis underlies HCC in more than $80 \%$ of all HCC patients (Fattovich et al., 2004). It has been suggested that any agent that leads to cirrhosis, may be considered as risk factors for the development of HCC. This risk is enhanced in those patients whose sera have increased levels of AFP (Idrees, 2008). There were 15 (8.37\%) cases that were caused by past HCB infection and relapsed to HCV infection. Two of them died even when they were receiving interferon therapy. All others HCC patients were chronic HCV patients.

HCV genotyping of the 179 patients was performed using standard technique (Idrees, 2008). In this study, we observed preponderance of infection by genotype 3 $(71.98 \%)$ among the rural patients in Faisalabad. The HCV genotype most commonly found throughout most of the urban population in Lahore, northern areas, southern and western areas of country was also found as genotype 3 , with percentages varying from 71.0 to maximum of $90 \%$ (Ahmed et al. 2007; Hakim et al. 2008; Hamid et al. 2004; Waheed et al. 2009). These authors reported that $51 \%$ of HCV patients had genotype $3 a ; 24 \%$ patients had a coinfection of $3 \mathrm{a} / 3 \mathrm{~b}$ and $16 \%$ predominantly had $3 \mathrm{~b}$. Afridi et al. (2009) also reported $50 \%$ preponderance of genotype $3 \mathrm{a}$ followed by $3 \mathrm{~b}$ and $1 \mathrm{a}$. The most exhausttive study of HCC was conducted by Idrees et al. (2009) on urban HCC patients. They found that $40.9,15.66,9.63$ and $2.4 \%$ among the $145 \mathrm{HCC}$ patients had $3 \mathrm{a}, 3 \mathrm{~b}, 1 \mathrm{a}$ and $1 \mathrm{~b}$, respectively. Based on these results and those of our study, we can conclude that most hepatitis C infections in Faisalabad region's rural population were due to genotype 3, with the highest proportion (80\%) also found in Bahawalpur (Qazi et al. 2006). This result implies that patients infected with HCV in this area should preferentially be treated with a specific clinical protocol for genotype 3 after confirmation.

\section{Conclusions}

The identification of chronic HCV infection is a major risk 
factor for the spread of HCC in Pakistan as already been reported for Italy, Japan and Spain. All these HCC cases are HCV-related and no case was linked with other types of viruses. HCV genotype 3a was the predominant genotype in $55.3 \%$ cases and is a major risk factor for the development of HCC and may cause increased oncogenecity in our population. HCC was linked with liver cirrhosis (81\%). It was also found that treatment with interferon did not stop the progression of HCC. It demands immediate attention of the concerned authorities to take maximum proper preventive measures to stop its transmission and progression in the general population of the country.

\section{ACKNOWLEDGEMENTS}

These studies were supported by GC University, Faisalabad and PINUM. Pakistan Atomic Energy Commission of Pakistan and Higher Education Commission, Pakistan provided all the equipments and chemicals/kits. The technical staff provided technical assistance. Ethical Committee is thanked for approving this study.

\section{REFERENCES}

Afridi S, Naeem M, Hussain A, Kakar N, Babar ME, Ahmad J (2009). Prevalence of hepatitis $\mathrm{C}$ virus (HCV) genotypes in Balochistan. Mol. Biol. Rep. 36: 1151-1154.

Ahmed N, Asgher M, Shafique M, Qureshi JA (2007). An evidence of high prevalence of hepatitis $C$ virus in Faisalabad, Pakistan. Saudi Med. J. 28: 390-395.

Bukhari SA, Rajoka MI, Nagra SA, Ur-rehman Z (2010). Plasma homocysteine and DNA damage profiles in normal and obese subjects in the Pakistani population. Mol. Biol. Rep. 37: 289-295

Farshapour F, Makvandi M, Samsarbafzadeh AR, Jalalifar MA (2010). Determination of hepatitis $C$ virus genotypes among blood donors in Ahvaz, Iran. Ind. J. Med. Microbiol. 28: 54-56.

Fattovich G, Stroffolini T, Zagni I, Donato F (2004). Hepatocellular carcinoma in cirrhosis: incidence and risk factors. Gastroenterology, 127: S35-S50.
Frank C, Mohamed MK, Stickland GT, Lavanchy D, Arthur PR, Magder LS (2000). The role of parenteral antischistosomal therapy in the spread of Hepatitis C virus in Egypt. Lancet. 355: 887-891.

Hakim ST, Kazmi SU, Bagasra O (2008). Seroprevalence of hepatitis B and $C$ genotypes among young apparently healthy females of Karachi-Pakistan. Libyan J. Med. AOP: 071123.

Hamid S, Umar M, Alam A, Siddiqui A, Qureshi H, Butt J ( 2004). Pakistan Society of Gastroenterology. PSG consensus statement on management of hepatitis C virus infection-2003. J. Pak. Med. Assoc. 54:146-150.

Higuchi M, Tanaka E, Kiyosawa K (2002). Epidemiology and clinical aspects on hepatitis C. Jpn. J. Infect. Dis. 55: 69-77.

Idrees M (2008). Development of an improved genotyping assay for the detection of hepatitis $\mathrm{C}$ virus genotypes and subtypes in Pakistan. J. Virol. Methods, 105: 50-56.

Idrees M, Rafique S, Rehman IU, Akbar H, Yousaf MZ, Butt S, Awan Z, Manzoor S, Akram M, Aftab M, Khubaib B, laz-uddin S (2009). Hepatitis C virus genotypes $3 a$ infection and hepatocellular carcinoma: Pakistan experience. World J. Gasttroenterol. 15: 50805085.

Kao JH, Chen DS (2002). Transmission of Hepatitis C virus in Asia: past and present perspectives. J. Gastroent. Hepatol. 15: 91-96.

Qazi MA, Fayyaz M, Chaudhrty GM, Jamil A, Malik AH, Gardezi Al, Bukhari MH (2006). Hepatitis C virus genotypes in Bahawalpur. Biomedical, 22: 51-54.

Ryu SH, Fan X, Xu Y, Elbaz T, Zekri AR, Abdelaziz AO, Di Bisceglie AM (2009). Lack of association between genotypes and subtypes of $\mathrm{HCV}$ and occurrence of hepatocellular carcinoma in Egypt. J. Med. Virol. 81: 844-947.

Simmonds P, Bukh J, Combet C, Deléage G, Enomoto N, Feinstone S, Halfon P, Inchauspé G, Kuiken C, Maertens G, Mizokami M, Murphy D, Okamoto H, Pawlotsky J, Penin F (2005). Consensus proposals for a unified system of nomenclature of Hepatitis $C$ virus genotypes. Hepatology, 42: 962-973.

Tavares NJ, Almeida D, Soares MC, Uchoa R, Viana S, Darub R, Farias E, Rocha G, Vitvitski L, Paraná R (2004). Seroprevalence of hepatitis $B$ and $C$ in the western Brazilian Amazon Region (Rio Branco, Acre): a pilot study carried out during a hepatitis B vaccination program. Braz. J. Infect. Dis. 8: 133-139.

Tong CY, Khan R, Beeching NJ, Tariq WU, Hart CA, Ahmad N (1996). The occurrence of hepatitis $B$ and $C$ viruses in Pakistani patients with chronic liver disease and hepatocellular carcinoma. Epidemiol. Infect. 117: 327-332.

Waheed Y, Shafi T, Safi SZ, Qadri I (2009). Hepatitis C virus in Pakistan; A systematic review of prevalence, genotypes and riskfactor. World J. Gasttroenterol. 15: 5647-5653. 